\title{
La importancia de las relaciones en la práctica profesional docente. Un estudio de casos
}

\author{
Gabriel R. Soto, Nelson T. Villagra \\ y Franco Correa
}

\section{Resumen}

El conocimiento matemático profesional que los profesores de matemática utilizan en su tarea se basa en las relaciones entre la matemática de la formación y la matemática a enseñar en el aula. Tales relaciones, surgidas en diferentes momentos de la formación, son utilizadas a menudo por los docentes para establecer jerarquías entre los conceptos matemáticos que se enseñan en la escuela. Es importante entonces identificarlas para poder diseñar e implementar dispositivos de formación que permitan a los profesores de matemática, en formación o en ejercicio, profundizar y fortalecer sus saberes profesionales. Presentamos un método de identificación y cuantificación de tales jerarquías, basado en las nociones de centralidad en redes sociales, para explicitar las relaciones entre los conceptos matemáticos que los docentes tienen a disposición para enseñar y compararlas con la matemática de su formación.

Palabras clave: desarrollo profesional de profesores de matemática, conocimiento matemático para enseñar, formación matemática de docentes, redes sociales.

Keywords: professional development of mathematics teachers, mathematics knowledge for teaching, mathematic training for teachers, social networks.

\section{Abstract}

The professional mathematical knowledge that mathematics teachers use in their work is based on the relations between mathematics of training and mathematics to be taught in the classroom. Such relationships, arising at different times of formation, are often used by teachers to establish hierarchies among the mathematical concepts taught in the school. It is important then, to identify them in order to design and implement training devices that allow mathematics teachers, in training or in practice, to deepen and strengthen their professional knowledge. We present a method of identification and quantification of such hierarchies, based on the notions of centrality in social networks, to make explicit the relationships between the mathematical concepts thatteachers have available to teach and compare them with the mathematics of their education. 
Autores: Soto, Gabriel Rubén (1)(2); Villagra, Nelson Tomás(1)(2); Correa, Franco Ignacio(2)

\section{Institución:}

(1) Facultad de Ciencias Naturales. Universidad Nacional de la Patagonia San Juan Bosco.

(2) Facultad Ingeniería. Universidad Nacional de la Patagonia San Juan Bosco.

Dirección: Ciudad Universitaria. Ruta Provincial $N^{\circ} 1$ - Comodoro Rivadavia (9005) Chubut

TE: +54 2974550836 - int 24 - int 29.

Dirección electrónica: gsoto@unpata.edu.ar 


\section{Introducción}

Definir buenas prácticas de enseñanza es una tarea compleja pues, entre otros aspectos, se deben caracterizar las decisiones que los docentes realizan respecto a qué enseñar, cómo enseñar y por qué enseñar. Lee Shulman (1987) propuso como alternativa de análisis de las prácticas de enseñanza, focalizar en el rol del contenido disciplinar en la tarea docente para identificar los modos en que los docentes usan sus conocimientos sobre el contenido, para transformarlos en representaciones didácticas útiles para enseñarlo y poder así desarrollar marcos de análisis acerca del conocimiento didáctico del contenido, de ahora en más CDC (Bolivar, 2005, p. 2). Esto es, para enseñar es necesario no sólo conocer definiciones y conceptos sino que, además, es preciso comprender los principios organizativos y estructurales del campo disciplinar, como así también qué ideas o conceptos son centrales en la disciplina y cuáles son secundarios, es decir, entender la matemática desde un punto de vista avanzado (Klein, 2016a, 2016b, 2016c). Siguiendo las categorías originales de análisis del CDC que presenta Shulman (1987, p.8), Debora Bally colaboradores extienden esta noción y presentan un modelo para el Conocimiento Matemático para la Enseñanza (MKT por sus siglas en inglés), en el cual incorporan dominios de análisis no sólo del CDC sino también para el conocimiento matemático disciplinar necesario para enseñar matemática (Ball, Thames y Phelps, 2008, p. 403). Si bien este modelo se convierte en una herramienta de análisis sobre cómo el contenido disciplinar y la metodología interactúan en el desarrollo e implementación de dispositivos de desarrollo profesional docente (cursos de formación, diseños curriculares, libros de textos, etc.), no siempre es posible identificar en el análisis las condiciones sociales en las cuáles los participantes de tales dispositivos se desarrollan y los condicionamientos que ejercen sobre estas prácticas las políticas educativas gubernamentales (Silvy et al, 2013, Artigue, 2012). Particularmente, resulta de suma importancia analizar cómo los docentes movilizan sus saberes matemáticos en un contexto de enseñanza (Proulx y Berdnaz, 2009), siendo ésto un tema central en los programas de formación docente, pues como afirman Olbrich, Gualdoni y Meshler (2015) existe evidencia que la matemática que aprenden en su formación está desconectada de la matemática que tienen que enseñar en la escuela puesto que la construcción de esta última pareciera quedar a cargo de los estudiantes de profesorado en Matemática tanto en institutos de formación docente como universidades nacionales. Más aún, los docentes desarrollan durante sus trayectos de formación (inicial, continua, incluso en sus propias prácticas) saberes experienciales a través de los cuales dominan su profesión y, en vista que en general no participan en la toma de decisiones curriculares sobre los contenidos presentes en su formación, estos saberes pueden distanciarse de los adquiridos fuera de esos trayectos formativos (Tardiff, 2004, pag. 37).

Resulta entonces que en cada instancia de formación coexisten, potencialmente, dos redes conceptuales de contenidos matemáticos que coexisten en constante tensión: una a la que llamaremos Red de Contenidos Matemáticos (RCM), surgida a partir del desarrollo de la matemática como campo disciplinar de la comunidad matemática (Burton, 2009, págs. 159-160) que sustenta qué contenidos matemáticos se deben enseñar en la escuela y cómo se relacionan entre sí. La otra red conceptual de contenidos matemáticos es subjetiva y surge de la experiencia que los docentes adquieren durante sus trayectos formativos y de práctica, a la que denominaremos Red Matemática Docente (RMD). Si bien las redes mencionadas se construyen a partir de los mismos contenidos, ambas pueden diferenciarse en cómo estos últimos están relacionados entre sí, y son estas relaciones las que nos interesan poder estudiar. Se hace necesario entonces desarrollar herramientas que permitan analizar las tensiones que existen entre la redes RCM y la RMD. En este trabajo, encuadrado en el proyecto de investigación Aplicaciones de la teoría de redes al desarrollo de dispositivos de formación para docentes en matemática, financiado por la Secretaría de Ciencia y Técnica de la Univer- 
sidad Nacional de la Patagonia San Juan Bosco y cuyo objetivo principal es:

Construir conocimiento sustantivo que aporte a la retroalimentación de la formación disciplinar de profesores de Matemática, a sus prácticas profesionales y al desarrollo de dispositivos de formación tanto a profesores de matemática en formación como a los profesores de matemática en ejercicio de la región de influencia de nuestra universidad.

Presentamos resultados de un trabajo de comparación de las características estructurales y jerárquicas de redes conceptuales construidas por formadores de formadores, docentes de matemática en ejercicio y en formación durante diferentes instancias de formación durante el año 2015, que nos permitieron reflexionar acerca del diseño e implementación de dispositivos de formación.

\section{Aspectos metodológicos para la construcción de las redes RCM y RMD}

Para la construcción de la red de contenidos matemáticos (RCM) y la red matemática docente (RMD) analizadas en el presente trabajo, utilizamos como modelo de RCM la red conceptual presentada por Solomon (1987, p. 21), como puede observarse en el panel izquierdo de la Figura 1. La elección de esta red no ha sido del todo arbitraria, pues ha sido utilizada como referencia en textos relacionados con la formación docente (Fiol y Fortuny, 1999). Para la obtención de las RMD sólo se utilizaron las representaciones gráficas de los contenidos matemáticos incluidos en la red de Solomon por considerarlos estándares y así minimizar la intervención del equipo de investigación al momento de implementar la encuesta, siendo los mismos los nodos o vértices de las redes RCM y RMD.

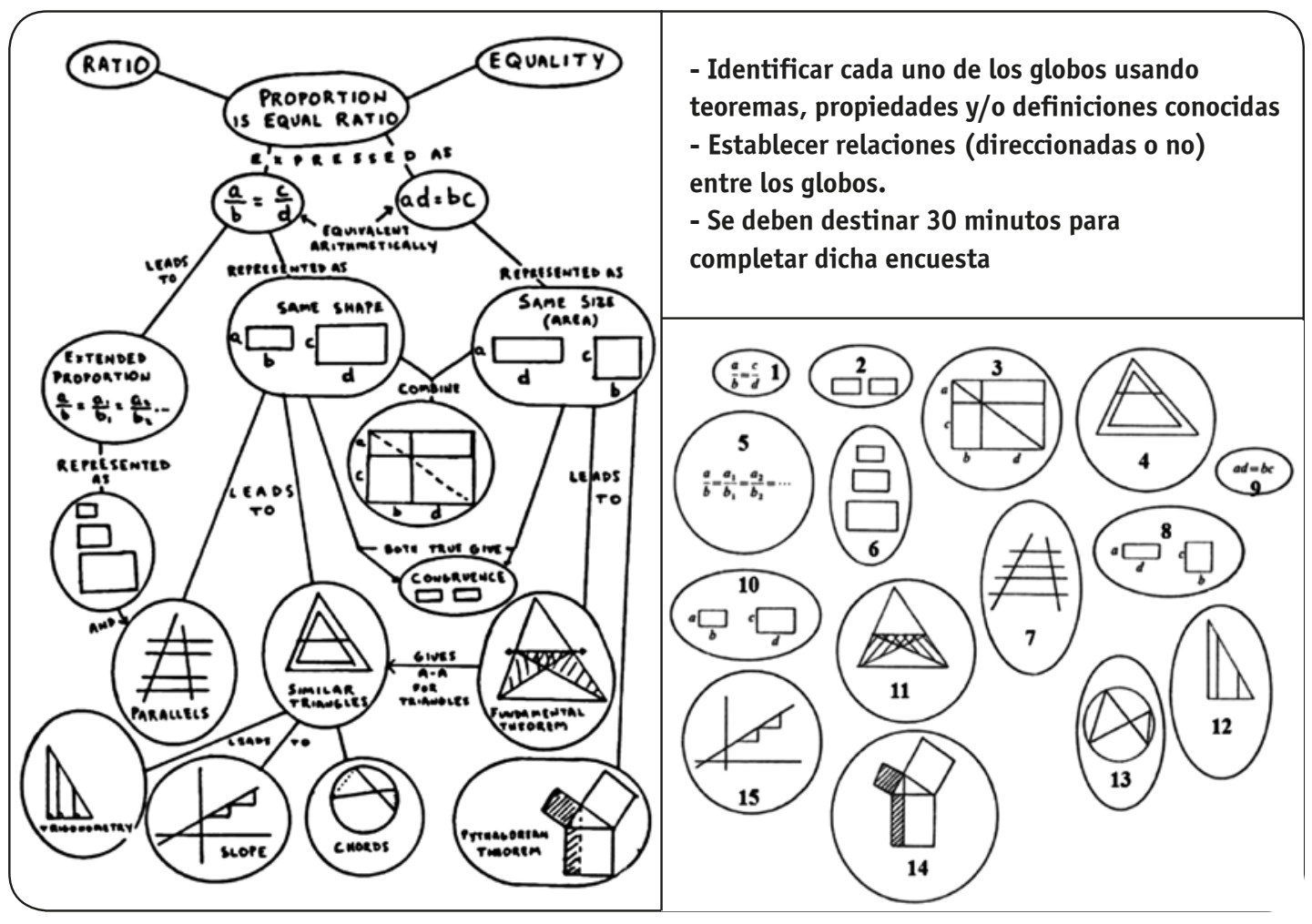

Figura 1.

Panel izquierdo: ed conceptual de Solomon (1987). Panel derecho: Encuesta presentada a los docentes. La enumeración de los conceptos (nodos o vértices) es arbitraria y es utilizada a lo largo de este trabajo. 
Se encuestaron a noventa y dos docentes, entre los cuales había profesores de enseñanza primaria y secundaria en ejercicio, estudiantes de profesorado en Matemática (tanto de institutos de formación docente como de universidades nacionales) y formadores de formadores de la ciudad de Comodoro Rivadavia y Esquel (Chubut, Argentina) y de la ciudad de San Salvador (El Salvador), durante el desarrollo de diferentes dispositivos de formación docente en los cuales los participantes tenían treinta minutos para construir todas las relaciones que ellos consideraban posibles entre los diferentes contenidos matemáticos presentados.

\section{Aspectos metodológicos para el análisis de las redes RCM y RMD}

Para realizar la comparación entre las redes RCM y RMD se eligieron dos parámetros de centralidad en redes sociales, intermediación y cercanía, considerados como indicadores de selección y/o agrupamiento de tareas para resolución de tareas (Freeman, 1978, p.216).

La intermediación $(B C$, betweenness centrality su nombre en inglés) es la frecuencia con que un vértice es parte de posibles caminos óptimos, llamados geodésicas, entre dos nodos cualesquiera diferentes del dado. Este parámetro es un indicador de qué tan importante puede ser considerado un concepto matemático como paso intermedio en un posible camino que une otros dos cualesquiera. De esta manera, un concepto que es parte de caminos diferentes, que enlazan cualesquiera otros dos se convierte en central en el sentido de que si lo eliminamos junto con todas las conexiones que convergen a él, efectivamente aumenta la distancia entre dos conceptos cualesquiera, y el «tiempo de enseñanza» se prolonga. Este parámetro se puede utilizar para determinar cuáles conceptos son centrales al momento de organizar y/o planificar por ejemplo una secuencia de enseñanza.

La cercanía (CC, closeness centrality su nombre en inglés) es la frecuencia con que un vértice de la red se relaciona directamente con el resto de los nodos. La cercanía mide la propiedad que tienen algunos conceptos de relacionarse con el resto de una manera directa. Si eliminamos algún concepto, junto con todas sus conexiones, con cercanía alta, estamos al igual que en el caso de intermediación, extendiendo la distancia entre otros nodos de la red.

Los parámetros de intermediación (BC) y cercanía (CC) son números definidos entre 0 y 1 y fueron calculados para cada uno de los nodos de las redes RCM y RMD utilizando el software abierto y libre Pajek (de Nooy et al., 2013). 


\section{Resultados descriptivos}

La RCM resultó conexa, siendo los nodos 7 y 10 , correspondientes a la representación clásica del Teorema de Thales y de rectángulos semejantes, los que obtuvieron los parámetros de intermediación y cercanía más altos. Para establecer una comparación entre las RCM y las RMD obtenidas a partir de las encuestas, se promediaron valores de los parámetros de intermediación y cercanía de cada red conceptual, resultando las mayores diferencias en los nodos 7 y 10. Esto es, los conceptos de proporcionalidad de segmentos y cuadriláteros no tienen la misma jerarquía promedio en las RMD respecto a la RCM. Además de la jerarquización de nodos en términos de cercanía e intermediación, obtuvimos una red promedio como un modelo para la RMD. Para ello, dado que cada red tiene su matriz de adyacencia correspondiente, calculamos el promedio entre ellas y determinamos que si una relación dada entre dos nodos debe ser incluida en la red promedio, tiene que tener un valor promedio mayor a 0,5. Las relaciones de la red promedio se muestran en el panel izquierdo de la Figura 2.
Es de destacar que la relación más frecuente entre las redes RMD fue entre el nodo 1 y 9 ; ocurrió setenta y nueve veces, siendo la base de esta relación el pasaje de términos que permite concluir que ambas expresiones son equivalentes. Además, la red promedio de las RMD aparece una relación entre los nodos 8 y 20 que no está presente en la red RCM. En el panel derecho de la Figura 2, se muestra la distribución de relaciones en aquellas redes que contenían la relación entre los nodos 1 y 9 , observando que sólo 6 de las 79 redes tuvieron todas las relaciones correspondientes a la red promedio. Este análisis descriptivo nos permitió concluir que la red conceptual que los docentes disponen para su potencial utilización en un contexto de formación o de enseñanza, dista significativamente de la que actualmente se proponen construir en los dispositivos de formación, sin lograr explicitar cómo las relaciones de la RCM pueden ser útiles al momento de desarrollar y/o potenciar sus saberes profesionales.

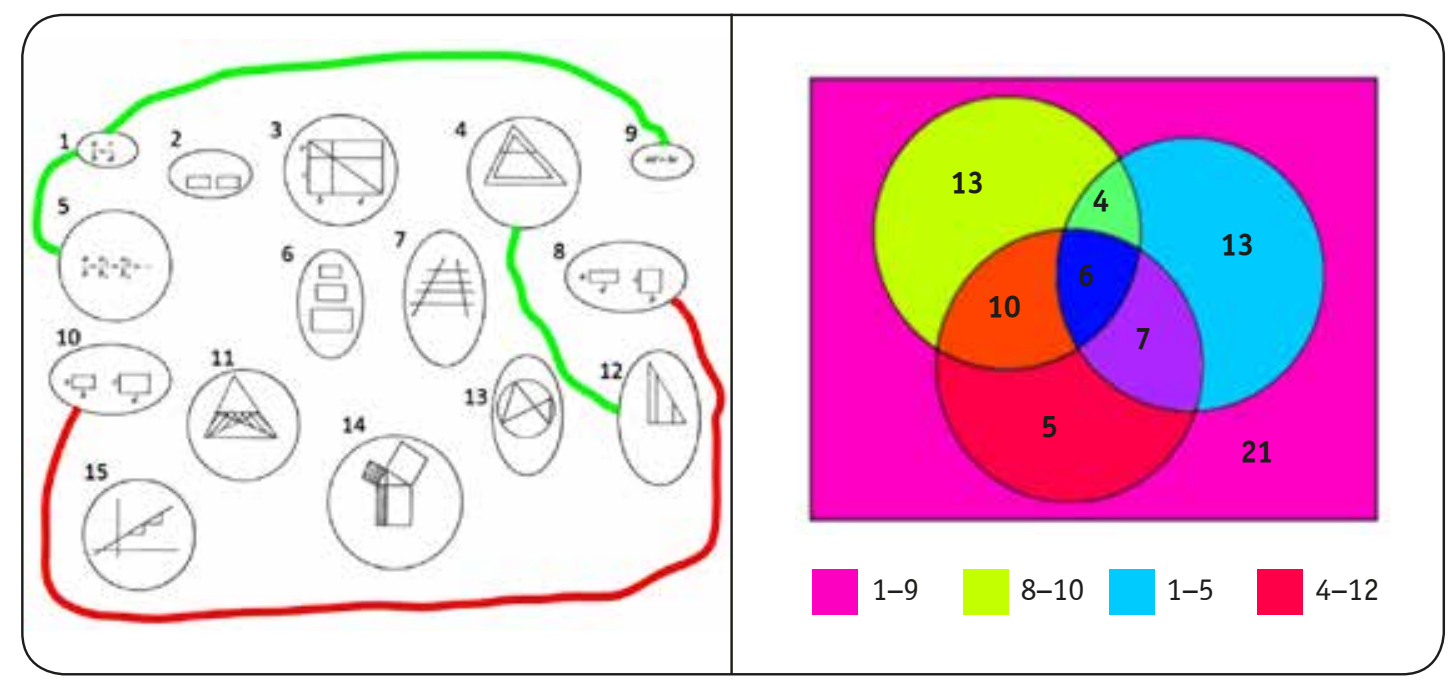

Figura 2.

Panel Izquierdo. Red promedio utilizada como modelo de RMD, obtenida a partir de considerar cuáles relaciones fueron las más frecuentes. Panel derecho: distribución de relaciones ente aquellas redes que contenían la relación entre los nodos 1 y 9. 


\section{Resultados por \\ agrupamiento de datos}

Luego de obtener las primeras diferencias cuantitativas entre la RCM y la RMD, nos preguntamos si era posible determinar, a partir de las matrices de intermediación y cercanías, si los datos obtenidos tenían alguna estructura de agrupamiento. Para ello, utilizamos el método de clusterización por k-means (Hartigan y Wong, 1979), el cual está predefinido en el paquete estadístico $\mathrm{R}$ (Kabacoff, 2011). Dada las características de nuestros encuestados, supusimos que tales datos podrían formar tres grupos, correspondientes a docentes en ejercicio, docentes en formación y formadores, cuyos resultados se muestran en la Figura 3.

Cada encuestado tiene una etiqueta $\mathrm{G}$ seguida de un número, y la etiqueta SOL corresponde a los parámetros de la red RCM. En ambos casos se observa que más del $90 \%$ de los encuestados forman un grupo diferenciado del resto. El $10 \%$ restante forman dos grupos, conteniendo la RMD en alguno de ellos y todas las respuestas de los formadores obtenidas.

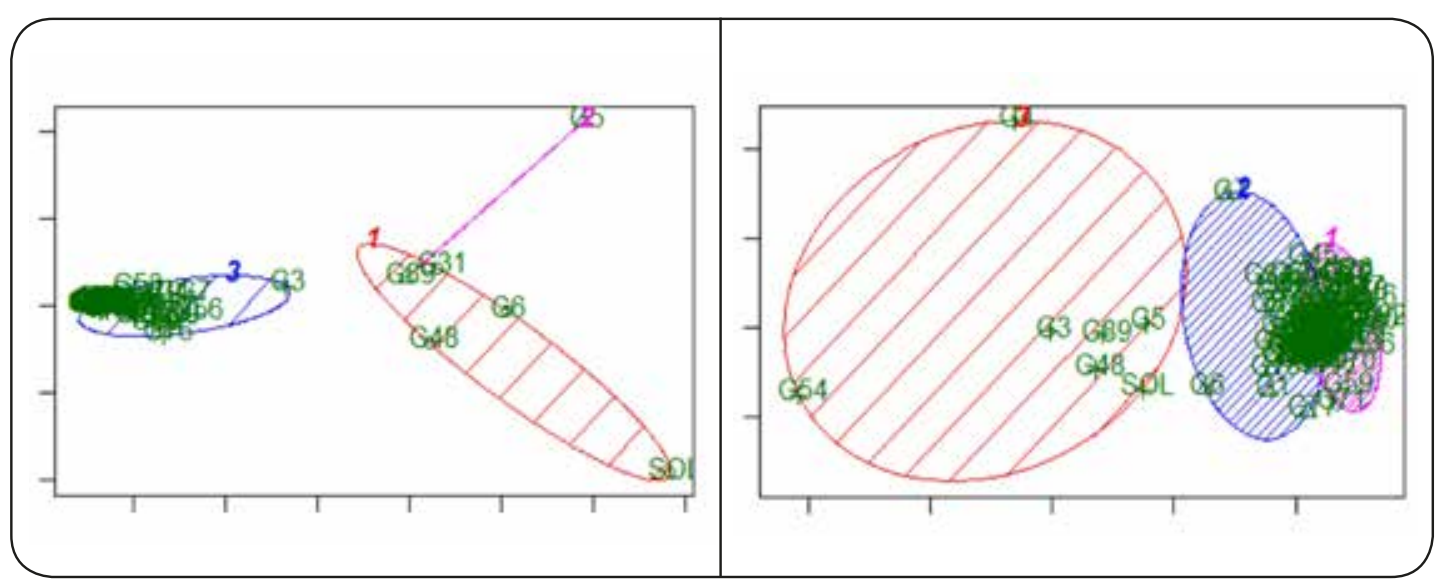

Figura 3.

Panel izquierdo: agrupamientos de los encuestados según parámetro de intermediación. Panel derecho: agrupamiento de encuestados según parámetro de cercanía. 


\section{Resultados sobre \\ dispositivos de formación}

En vista de estos resultados obtenidos, nos preguntamos si era posible utilizar esta metodología para analizar cuantitativamente la incidencia de un dispositivo de formación en la construcción de relaciones entre contenidos matemáticos. Para ello se encuestaron 17 profesores de El Salvador que participaron en un curso de formación docente de 30 horas de duración basado en un libro que incluía todos los contenidos representados en la RMD, en el cual se priorizaron las relaciones entre los contenidos incluidos en la RCM (Soto, 2015b). Los docentes completaron la encuesta descrita en el presente trabajo (panel derecho de la Figura
1) al inicio y al final del dispositivo de formación. Durante el desarrollo del curso se abordaron dichos contenidos, aunque en ningún momento se hizo mención específica a los mismos. Para la cuantificación se calcularon los parámetros de intermediación y cercanía para las redes completadas por cada docente, antes y después del curso, según se muestra en la Figura 4. Como puede observarse, las RMD mostraron, en promedio, un aumento general, lo que refleja que dichas redes se enriquecieron en cuanto a las relaciones (re)descubiertas por los docentes durante el dispositivo.

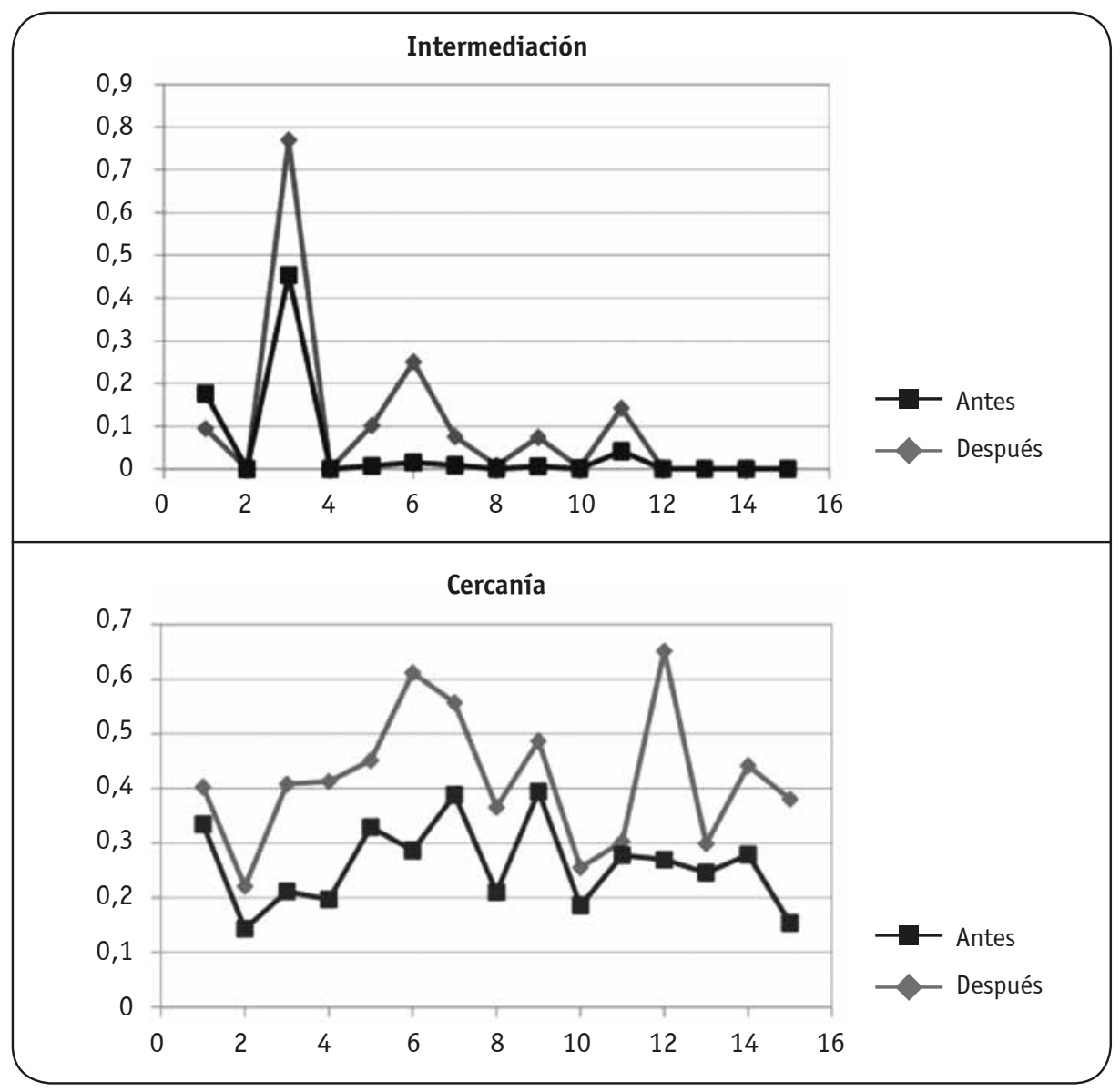

Figura 4.

Panel de arriba: intermediación promedio $(B C)$ de cada nodo de las redes RMD obtenidas, antes y después del curso de formación. Panel de abajo: dem para cercanía (CC). 


\section{Conclusiones}

Los saberes matemáticos que los docentes ponen en acción en una situación de enseñanza o aprendizaje se modifican, dependiendo de las interacciones que tengan con otros grupos profesionales; pueden resultar diferentes de los saberes matemáticos construidos por la comunidad matemática y que forman parte de la formación docente. Esta formación es un proceso individual que depende de las condiciones de lugar, tiempo y relaciones con la realidad que rodean una instancia de formación (Ferry, 2004). Por tanto resulta de suma importancia, al momento del diseño en implementación de dispositivos de formación, poder incorporar las construcciones subjetivas que los actores involucrados en ellos tienen para desarrollarse satisfactoriamente en su profesión (Clot y Fernández, 2007 p. 16). En el presente trabajo presentamos una herramienta de análisis cuantitativo que nos ha permitido identificar estas construcciones subjetivas al momento de reflexionar sobre las relaciones existentes entre contenidos de matemática a enseñar en la escuela. La utilización de ideas de centralidad provenientes de la teoría de redes mediante el uso de los parámetros de intermediación y cercanía nos han permitido tener una idea de cómo los docentes en formación, en ejercicio y los formadores, construyen el conocimiento matemático para enseñar a través de las relaciones entre contenidos matemáticos a enseñar. Los resultados obtenidos nos han mostrado que las relaciones entre los contenidos matemáticos a enseñar en la escuela son diferentes entre el grupo de formadores de formadores y los docentes, tanto en formación como en ejercicio, siendo esto una confirmación de la discontinuidad en la formación descrita hace más de cien años por F. Klein en 1908 (Klein, 2016a, p. 1). Nuestro análisis cuantitativo nos ha mostrado la desconexión subyacente en la forma en que los docentes construyen el conocimiento matemático para enseñar: la mayoría de las redes obtenidas en la encuesta resultaron desconexas. Esto podría ser un indicador de la fragmentación que existe en muchos de los programas curriculares y/o dispositivos de formación entre la matemática a enseñar en la escuela y la matemática que forma parte del conocimiento matemático para enseñar. Estos resultados evidencian que la matemática de la formación no es percibida por los docentes como un campo disciplinar coherente e interrelacionado, en corcondancia con los resultados cualitativos de la investigación desarrollada por Olbrich y otros (Olbrich, Gauldonia y Mesher, 2015). En términos de la red promedio de las RMD aparecieron relaciones ausentes en la red RCM (relación entre los nodos 8 y 20, panel izquierdo de la Figura 2), mostrando la incidencia que pueden tener los saberes experienciales (Tardiff, 2004) y el género de la actividad docente (Fernández y Clot, 2007) en el conocimiento matemático para enseñar. La identificación de estas relaciones diferentes tiene la potencialidad de poder hacer foco en cuestiones inherentes al campo matemático incluido en la formación docente.

En términos de la incidencia de dispositivos de formación en la práctica docente, esta herramienta permitió determinar el efecto que tiene un curso de desarrollo profesional en términos de las relaciones entre los conceptos de una red conceptual. En particular, para este dispositivo, se observó un aumento en los parámetros de intermediación y cercanía, el cual significa que las relaciones entre los conceptos matemáticos incluidos en las RMD aumentaron.

Las herramientas de análisis cuantitativo presentadas en este trabajo tienen la potencialidad de poder focalizar en aspectos y problemáticas inherentes a la formación de docente en matemática tales como qué matemática hay que enseñar en la formación, qué matemática tiene que aprender los docentes, cuál es el rol del eje de formación disciplinar en la formación docente, entre otros. La utilización de herramientas de la teoría de redes nos permitirá obtener perspectivas de análisis, complementarias a las existentes, respecto a las condiciones que se ofrecen a los docentes para llevar adelante su formación. 
Agradecimientos: Queremos agradecer a M. González, M. G. Mendonca, L. Da Luz Pereira, E. Gómez, L. Díaz y C. Negrette por sus aportes e ideas sobre este manuscrito, como asítambién a las observaciones realizadas por los evaluadores. Este trabajo ha contado con el apoyo de la Secretaría de Ciencia y Técnica de la Universidad nacional de la Patagonia San Juan Bosco.

\section{Referencias bibliográficas}

Artigue, M. (2012) Challenges in basic mathematics education. UNESCO.

Ball, D. L.; Thames, M.H. \& Phelps, G. (2008). Content Knowledge for teaching. What makes it special? Journal of Teacher Education, 59 (5), 389-407.

Bolivar, A. (2005) Conocimiento didáctico del contenido y didácticas específicas. Profesorado. Revista de currículum y formación del profesorado. 9 (2) 1-37

Burton, L. (2009) The Culture of Mathematics and the Mathematical Culture. En 0. Skovsmose et al. (Eds.) Universidad de Ciencias y Matemáticas Educación en Transición (157-173). Springer Science + Business Media LLC.

De Nooy, W.; Mrvar, A. \& Bataglej, V. (2005) Exploratory Social Network Analysis with Pajek. Cambridge University Press.

Fernández, G. y Clot, Y. (2007). Entrevistas en auto-confrontación: un método en clínica de la actividad. Laboreal, 2 (1), 15-19.

Ferry, G. (2004) Pedagogía de la Formación. Novedades Educativas, 53-58.

Fiol, M.L. y Fortuny, J.M. (1999) Proporcionalidad directa. La forma y el número. Colección: Matemáticas: cultura y aprendizaje. Editorial Síntesis.

Freeman, L.C. (1978) Centrality in Social Networks. Conceptual Clarification. Social Networks, 1, 215-239.

Hartigan, W. (1979) A K-Means Clustering Algorithm Journal of the Royal Statistical Society. Series C (Applied Statistics), 28 (1), 100-08

Kabakoff, R. (2011) R in Action. Data analysis and graphics with R. Manning Publications.

Klein, F. (2016a) Elementary mathematics from a higher standpoint. Volumne I. Arithmetic, Algebra, Analysis. Springer-Verlag

- - (2016b) Elementary mathematics from a higher standpoint. Volumne II. Geometry. SpringerVerlag

-_- (2016c) Elementary mathematics from a higherstandpoint. Volumne III. Presicion Mathematics and Approximation Mathematics. Springer-Verlag

Ma, L. (2010) Knowing and teaching elementary mathematics. Teacher's understanding of fundamental mathematics in China and the United States. Taylor y Francis.

Olbrich, M.; Gualdoni, V. y Meshler, M. (2015) Los profesorados en Matemática de la región Patagónica: aportes del campo de la investigación a las prácticas de formación. En Flores, M (comp.) IV Jornada de intercambio de experiencias en la enseñanza de la Facultad de Ingeniería. Universidad Nacional de la Patagonia San Juan Bosco. Eitorial Universitaria Patagónica. Comodoro Rivadavia.

Proulx, J. y Bednarz, N. (2009) Quelle formation mathématique pour les futurs enseignants du secondaire? Un éclairage fondé sur une analyse des recherches. Proceedings of Espace Mathématique Francophone (EMF-2009). Dakar, Senegal.

Silvy, C., Delcroix, A. y Mercier, A. (2013) Enquête sur la notion de «pedagogical content knowledge», interrogée à partir du «site local d'une question», Éducation et didactique, 7 (1), 33-58.

Schoenfeld, A. (1998) Towards a theory of teaching in context. Issues in Education, 4 (1), 1-94. Shulman, L.S. (1987). Knowledge and teaching: Foundations of the new reform. Harvard Educational Review, 57 (1), 1-22. 
Solomon, A. (1987). Proportion: Interrelations and Meaning in Mathematics. For the learning of mathematics, 7 (1), 14-22.

Soto, G. (2015a) Matemática a enseñar para enseñar. El caso de las fracciones. XIV Conferencia Interamericana de Educación Matemática. Chiapas, México.

(2015b) (Des)-haciendo matemática: desde Pitágoras a Descartes. Editorial Universitaria Patagónica.

Strogatz, S. (2001) Exploring complex networks. Nature, 410, 268-276.

Tardiff, M. (2004) Los saberes del docente y su desarrollo profesional. Marid: Narcea. 